\title{
Stroboscopic and Multiparametric Acoustic Analysis of Voice after Vocal Loading Task
}

\author{
1'John Samuel, ${ }^{2}$ Shenbagavalli Mahalingam, ${ }^{3}$ Subramaniyam Balasubramaniyam \\ ${ }^{4}$ Prakash Boominathan, ${ }^{5}$ Ravikumar Arunachalam \\ ${ }^{1}$ Associate Professor, Department of ENT, Head and Neck Surgery, Sri Ramachandra University, Chennai, Tamil Nadu, India \\ ${ }^{2}$ Lecturer, Department of SLHS, Sri Ramachandra University, Chennai, Tamil Nadu, India \\ ${ }^{3}$ Assistant Professor, Department of SLHS, Sri Ramachandra University, Chennai, Tamil Nadu, India \\ ${ }^{4}$ Professor, Department of SLHS, Sri Ramachandra University, Chennai, Tamil Nadu, India \\ ${ }^{5}$ Professor and Head, Department of ENT, Head and Neck Surgery, Sri Ramachandra University, Chennai, Tamil Nadu, India
}

Correspondence: John Samuel, Associate Professor, Department of ENT, Head and Neck Surgery, Sri Ramachandra University Chennai, Tamil Nadu, India, e-mail: johnsam71@yahoo.co.in

\section{ABSTRACT}

Introduction: Voice overuse may result in roughness, fatigue and pain while speaking. Vocal loading tasks have been used to assess changes in voice quality under different controlled settings. This study was done to document changes in voice characteristics before, immediately and 24 hours post-vocal loading task (VLT) using stroboscopic and acoustic analysis.

Materials and methods: Ten healthy adult males (age range: 20 to 40 years) with no apparent comorbid illness participated in the study. They were instructed to read the standard Rainbow passage at intensity above $75 \mathrm{~dB} \mathrm{SPL}$ until they perceived any symptoms of fatigue/ strain. Stroboscopy and comprehensive voice assessment were done on all subjects before, immediate post VLT and 24 hours post VLT. Results: Symptoms of vocal fatigue were noted on an average of 45 minutes of loud reading. Vocal fold edema, ventricular band hyperadduction and arytenoid congestion were noticed in immediate post task. The vocal fold movements were asymmetric and aperiodic with reduced mucosal wave and amplitude. Maximum phonation time (MPT) revealed statistically significant decrease (approximately $7 \mathrm{sec}$ ). Multiparametric acoustic analysis revealed a statistically significant increase in fundamental frequency, perturbation measures and lowest intensity with significant decrease in dysphonia severity index (DSI). All parameters (stroboscopic findings, perceptual, aerodynamic and acoustic analysis) showed values within normal limits after 24 hours post-task indicating recovery at 24 hours after vocal loading. Stroboscopy served as an evidence for structural and functional changes in the vocal fold. Changes in voice characteristics and recovery following vocal loading task can be documented using comprehensive voice assessment.

Keywords: Stroboscopy, Multiparametric acoustic analysis, Vocal loading task.

\section{INTRODUCTION}

A pleasant voice quality is a prerequisite for effective communication. Vocally abusive behavior, erratic food habits and work pressure contribute to changes in voice quality. ${ }^{1}$ These changes in voice are most of ten ignored in the early stages. Use of voice in potentially strenuous conditions may lead to vocal fatigue. Titze (as cited in A ronson \& Bless ${ }^{1}$ ) reported physiological alterations after prolonged voice use and vocal fatigue. These physiological alterations resulted in changes in laryngeal and respiratory muscles, and tissue viscosity. These changes in muscles and viscoelastic property of the vocal fold may lead to alterations in voice.

$\checkmark$ ocal fatigue is a frequent complaint among professional voice users and in individuals with voice disorders. Symptoms of vocal fatigue include complaints of dryness in throat, increased effort to talk, inability to talk continuously, hoarse or breathy voice quality and a tired or weak voice. ${ }^{2} \mathrm{~V}$ ocal fatigue can be related to the inability of the laryngeal system to withstand prolonged phonation or vocal loading. K elchner et al ${ }^{3}$ defined vocal loading as "prolonged loud voice use and has four distinct phases: warm up (adapting to the voicing task), performance (continuance of the voicing task), vocal fatigue (increased physical effort while speaking), and rest or recovery". Prolonged loud reading is one of the vocal loading tasks that is most often used to mimic excessive usage of voice. ${ }^{3-5}$

Vocal loading tasks have been used to simulate vocal hyperfunction and the effects have been studied objectively in western population. In India, various studies had been carried out in assessing the acoustic and aerodynamic changes after vocal loading task. ${ }^{6,7}$ Though these studies provided valuable information on acoustic and aerodynamic changes, visual ization of laryngeal structures was not done. V isualization of laryngeal structures is essential in order to fill the gap of documenting and val idating the other voice assessment parameters. This study is designed on the model of applying vocal loading task for assessing vocal fold mobility and voice characteristics objectively using the stroboscope and multiparametric acoustic voice analysis.

\section{AIMS}

1. To objectively measure the changes in voice characteristics after vocal loading task in normal adult males using stroboscopy and multiparametric acoustic voice analysis. 
2. To document complaints associated with vocal fatigue and the recovery of voice characteristics after vocal loading, while the subjects were adopting vocal hygiene and conservative voice use.

\section{MATERIALS AND METHODS}

A pilot study to evaluate the changes in vocal fold following VLT was conducted at the tertiary care hospital in Chennai. Ten healthy adult males in the age range of 20 to 40 years consented to participate in the study. The subjects were recruited based on the exclusion and inclusion criteria given below:

\section{Exclusion Criteria}

1. History of smoking, laryngeal pathology, intubation, neurologic disorder, respiratory disorder, systemic illness, and previous surgery/accident/trauma to the larynx.

2. Sustained (prolonged use) medications for any medical condition.

3. Underweight or obesity.

\section{Inclusion Criteria}

1. Perceptually normal voice in terms of pitch, loudness and quality.

2. L oudness dynamic range of 40 to $80 \mathrm{~dB} \mathrm{SPL}$.

\section{Method}

A ll subjects underwent a basel ine voice assessment prior to the $V L T$. Then the subjects were asked to read a standard passage at a loud intensity up to an hour or until they reported fatigue. Immediately following VLT and 24 hours post VLT, voice assessment was done to document changes in vocal fold.

\section{PROCEDURE}

The study was carried out in three phases:

Phase I: Baseline measurement

Phase II: Vocal loading task

Phase III: Postvocal loading task.

\section{Baseline Measurement}

Sri Ramachandra voice assessment protocol developed by Boominathan et al ${ }^{8}$ was used for voice assessment. This protocol included information on demographic data, brief complaint, history on vocal and nonvocal habits and the parameters of voice assessed under the following:

1. Stroboscopy analysis: Stroboscope (ATMOS Medizin Technik $\mathrm{GmbH}$ (o) was used to obtain laryngeal images and was assessed by otolaryngologist and speech pathologist for structure and function. The parameters assessed were: glottal closure, regularity, symmetry of the vocal fold vibration, mucosal wave and amplitude of vocal fold vibration, nonvibratory portion (if any) and hyperadduction of ventricular band.
2. Perceptual analysis: The GRBAS scale ${ }^{9}$ was used for the perceptual analysis of voice for conversation tasks. The phonation and conversation samples were recorded for perceptual analysis. The samples were judged for the perceptual correlates of voice by an experienced speech pathologist for parameters of pitch, loudness and quality.

3. Aerodynamic analysis: The subjects were instructed to phonate/a/, /i/ and / $\mathrm{u} /$ for three trials and the maximum was taken as maximum phonation time (MPT), s/z ratio was al so calculated.

4. Acoustic analysis: L ingW aves phonetogram Pro and Signal A nalysis M odule (version 2.4) was used to measure DSI. Recording was done using a condenser microphone attached to a sound pressure level meter (Center 322) mounted on a tripod. It enabled adjustment of height and alignment of the mic to the subject's mouth. The inbuilt mic was covered by wind screen to reduce the sensitivity of mic to surrounding noise.

The subjects were instructed to sit in an upright, relaxed posture. M outh to mic distance was approximately $30 \mathrm{~cm}$. Following steps were used to obtain a phonetogram:

1. Phonation profile: The subjects were asked to sustain their singing scale and/or to sustain /a/ at four levels: L ow pitchlow intensity; low pitch-high intensity; high pitch-low intensity and high pitch- high intensity. The clinician initially model ed the subjects. The subjects were asked to phonate and samples were recorded.

2. Speaking profile: R eading a passage (R ainbow passage) or counting numbers at three intensity (soft, moderate and loud) levels each for minimum of 30 seconds was recorded.

Dysphonia severity index (DSI): DSI was calculated by the software using the formula given by $W$ uyts et al: ${ }^{10}$

$$
\begin{aligned}
\mathrm{DSI}=0.13 \times \mathrm{MPT}+ & 0.0053 \times \mathrm{F}_{0} \text {-high }-0.26 \times \mathrm{I}_{0} \text {-low }-1.18 \\
& \times \mathrm{jitter}+12.4
\end{aligned}
$$

\section{Vocal Loading Task}

The procedure was recorded using LingW aves phonetogram Pro and Signal A nalysis M odule (V ersion 2.4). A Sound Level $M$ eter (SLM) (Center 322) with a free field condenser microphone was mounted on a tripod stand and kept at a distance of $30 \mathrm{~cm}$ from the subject's mouth. The subjects were instructed to read the Rainbow passage at intensity above $75 \mathrm{~dB}$ SPL in standing posture (Fig. 1). The subjects were instructed to read up to an hour or until they reported themselves as fatigued. If a subject was unable to read at the instructed intensity level for 1 hour, the reading was terminated and the duration of actual reading was noted. In addition, the investigator observed and noted any physical or vocal signs of discomfort (e.g. coughing, throat clearing and voice quality change).

The subjects were cued for decreased intensity level by the indicator in the computer. The investigator monitored and noted the time of initial sign of vocal fatigue from the beginning of the reading. 


\section{Post-Task Phase}

All subjects underwent immediate and 24 hours post-task assessment following the vocal loading task. The subjects were asked to follow strict vocal hygiene guidelines after the immediate post-task assessments which included the following advise: (i) N ot to engage in vocally abusive behavior (yelling, prolonged singing, talking, whispering and throat clearing), (ii) to avoid caffeine, (iii) to avoid passive smoking, and (iv) to drink half glass of water for every 25 to 30 minutes when awake for 24 hours after the task. The 24 hours post-task measurements were recorded to document the recovery after 24 hours.

\section{Statistics}

Paired t-test was used to measure the pre- and post-task voice measures.

\section{RESULTS}

\section{Prolonged reading as Indicator of Vocal Fatigue}

The average duration of sustained reading of subjects was approximately 42 minutes (SD: 9.4 ) and ranged from 30 to 60 minutes (Fig. 2). Nearly $80 \%$ of the subjects complained of 'dryness of throat' and 'sensation to frequently swallow' at the

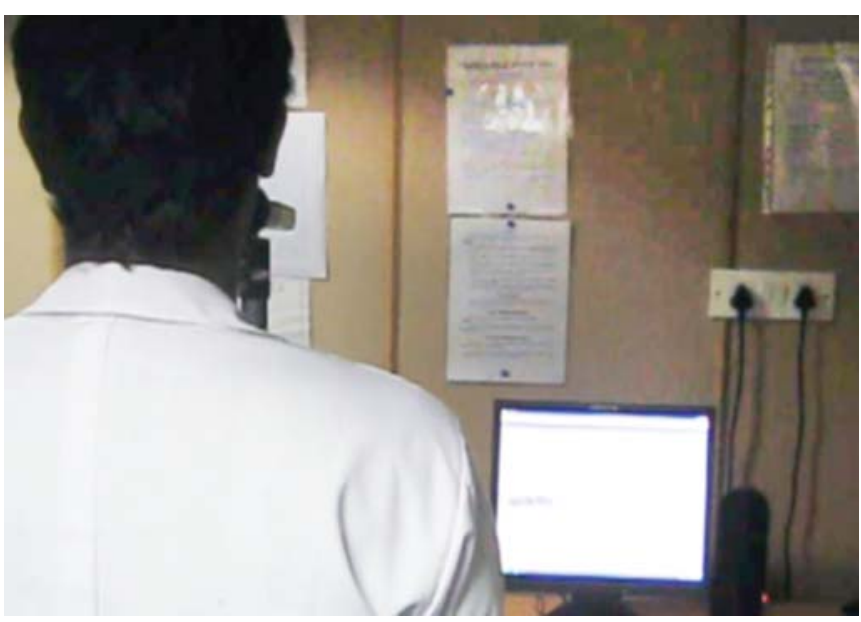

Fig. 1: Vocal loading task

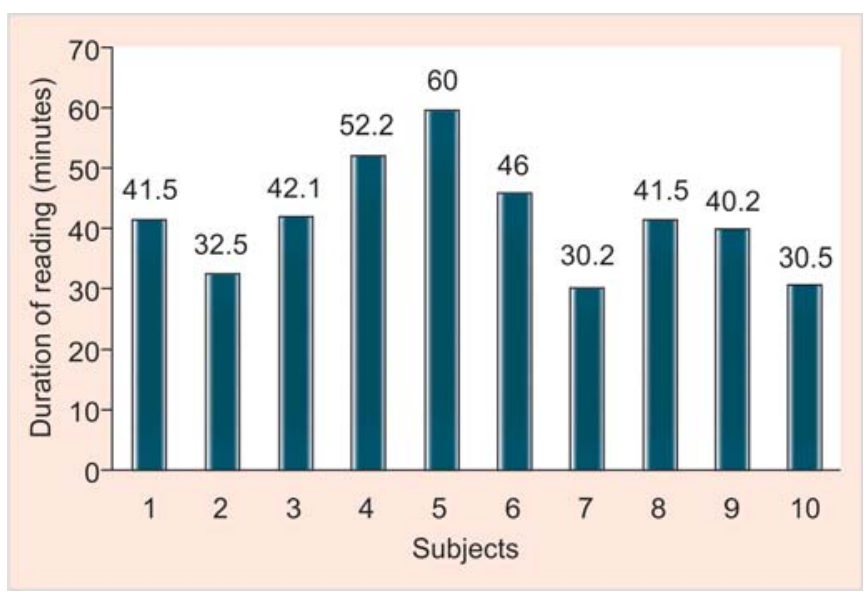

Fig. 2: Duration of reading of each subject



Fig. 3: Percentage of subjects showing vocal fatigue symptoms

end of vocal loading task. Change in voice was the least common vocal fatigue symptom reported. This could probably be attributed to the subject's insensitiveness to voice change (Fig. 3).

\section{Baseline vs Post-Vocal Loading Task Measurement}

Stroboscopic evaluation at baseline, immediate post and 24 hours post-VLT for all the subjects is tabulated in Table 1. Perceptual evaluation using GRBAS scale revealed overall grade to be normal in baseline measurement. In immediate postvocal loading task, all subjects showed slight to moderate deviation in overall grade. The mean of M PT (M ean: 23.1 seconds; S.D:1.2) and s/z ratio (M ean: 1.1; SD: 0.04) of the subjects in baseline measurement was within normal limits. In the immediate post-task, M PT showed an average decrease of 7 seconds (M ean: 15.5 seconds; SD : 2.4 ) for all the subjects, which was statistically significant $(p<0.01)$ and $s / z$ ratio showed a statistically significant increase (M ean 1.27 ; SD: 0.1 ).

In the baseline measurement, acoustic analysis revealed values within normal limits. In post-task phase, fundamental frequency $\left(F_{0}\right)$ of phonation on an average showed a significant increase of $14 \mathrm{~Hz}(S D: 2.4)(p<0.01)$ for all the subjects. The findings were similar to the studies done in the past which showed increase in fundamental frequency following vocal loading task. ${ }^{4,11}$ The perturbation measures showed a significant increase in the immediate post-task phase $(p<0.05)$. It was suggestive of irregular and aperiodic movement of vocal folds resulting in increased perturbation measures. The mean (SD) of lowest intensity $\left(\mathrm{I}_{0}\right)$ in baseline and post-task was found to be $53 \mathrm{~dB}$ SPL (4.5) and $60.29 \mathrm{~dB}$ SPL (7.2) respectively. There was an increase in the lowest intensity $\left(I_{0}\right)$ of approximately 7 $\mathrm{dB} S P L$ in all the subjects, which was statistically significant $(p<0.01)$. This indicated the effects of vocal fold edema and reduced pliability of the vocal folds for phonation in the immediate post-task phase. The need for greater subglottal pressure to set the vocal folds into vibration, results in inability to produce soft voice. 
Table 1: Baseline, immediate post-VLT and 24 hours post-VLT stroboscopic evaluation of all subjects

\begin{tabular}{llll}
\hline Stroboscopy parameters & Baseline measurement & Immediate post-VLT & 24 hours post-VLT \\
\hline Closure & Complete & Irregular & Complete \\
Symmetry & Symmetrical & Asymmetrical & Symmetrical \\
Periodicity & Periodic & Aperiodic & Periodic \\
Amplitude (Rt and Lt) & Normal & Reduced & Normal \\
Mucosal wave (Rt and Lt) & Normal & Reduced & Normal \\
Nonvibratory portion (if any) & None & None & None \\
Hyperadduction of ventricular band & Absent & Present & Absent \\
Other findings & Normal structures and functions & Vocal fold edema, arytenoid & No evidence of \\
& & congestion and edema; & hyperfunction and \\
& & laryngeal hyperfunction present & edema \\
\hline
\end{tabular}


Fig. 4: Average values of aerodynamic and acoustic parameters in all three phases 
The other parameter assessed was dysphonia severity index (DSI). I t is a single composite index which includes maximum phonation time (MPT), high $\mathrm{F}_{0}$, low $\mathrm{I}_{0}$ and $\mathrm{J}$ itter in percentage ranging from -5 to +5 . The mean (SD) of DSI in baseline and immediate post-task was found to be 2.6 (1.2) and 0.08 (1.4) respectively. This study showed a slight to moderate deviation in DSI in the immediate post-task phase which was statistically significant $(p<0.01)$. This index reflects the changes in M PT, low $\mathrm{I}_{0}$ and $\mathrm{J}$ itter values which were mentioned earlier.

The 24 hours post-task stroboscopy showed return of symmetric, periodic vocal fold movement with complete glottal closure and no evidence of laryngeal hyperfunction. The mucosal wave showed normal morphology and amplitude of movement. In 24 hours postvocal loading, the perceptual evaluation of overall grade was found to be normal grade in all subjects. The aerodynamic and acoustic parameters showed significant change from immediate post-task and all parameters were well within normal limits in 24 hours postvocal loading indicating a recovery of the tissue change (Fig. 4).

\section{CONCLUSION}

The study concluded the following:

1. The heal thy Indian adult male was able to sustain reading at level above $75 \mathrm{~dB}$ SPL on an average for 42 minutes in vocal loading task. Vocal strain thus produced caused measurable changes in vocal fold mobility as well as in aerodynamic and acoustic measures of phonation which explained the perceptual changes in voice quality in these subjects.

2. The commonest reported symptoms of vocal fatigue were frequent swallowing sensation, dryness of mouth and cough. These symptoms in an otherwise normal, heal thy adult male with vocally abusive behavior need to be identified and appropriate corrective measures instituted, before they lead on to vocal fold disorders.

3. Practice of vocal hygiene measures does cause improvement in vocal fold function and voice characteristics. The duration of vocal hygiene practices depend on the duration and intensity of vocally abusive behavior before they are identified for correction. In our study, practice of vocal hygiene measures for 24 hours after vocal loading reversed the changes in vocal fold mobility and voice characteristics.

The limitation of the present study is the small sample size. Future directions of the study can be focused on vocal loading in females and in professional voice users.

\section{REFERENCES}

1. A ronson AE, Bless DM. Clinical voice disorders. N ew Y ork: Thieme Publications 2009.

2. Smith E, Kirchner HL, Taylor M, Hoffman H, LemkeJH. Voice problems among teachers: Differences by gender and teaching characteristics. J V oice 1998;12:328-34.

3. Kelchner $L$, Toner $M$, L ee $L$. Effects of prolonged loud reading on normal adolescent male voices. Lang Sp Hear Service Schools 2006;37:96-103.

4. Stemple J, Stanley J, Lee L. Objective measures of voice production in normal subjects following prolonged voice use. J V oice 1995;9(2):127-33.

5. K elchner $L$, Lee $L$, Stemple $C$. Laryngeal function and vocal fatigue after prolonged reading in individuals with unilateral vocal fold paralysis. J V oice 2003;17:513-28.

6. Boominathan $P, R$ ajendran $A, M$ ahalingam $S$, G nanavel $D$. $V$ oice characteristics and recovery patterns in Indian adult males after vocal loading. J A IISH 2010;20(2):220-31.

7. K rishna GS, N ataraja NP. Suscepti bility criteria for vocal fatigue. J Indian Sp Hear Assoc 1995;14:11-14.

8. Boominathan P, Samuel J, Ravikumar A, Nagarajan R. Sri Ramachandra U niversity protocol for voice assessment. Paper presented at The 4th World V oice Congress; Sep 6-9, 2010 Seoul, South Korea.

9. Hirano M. Clinical examination of voice. N ew Y ork: Springer V erlag 1981.

10. W uyts $F L$, De B odt M S, M olenberghs $G$, Remacle $M$, H eylen $L$, M illet $B$, et al. The dysphonia severity index: A n objective measure of vocal quality based on a multiparametric approach. J Sp Lang Hear Res 2000;43:796-809.

11. Vilkman E, Lauri ER, Alku P, Sala E, Sihvo M. Effects of prolonged oral reading on $\mathrm{F}_{0}, \mathrm{SPL}$, subglottal pressure and amplitude characteristics of glottal flow waveforms. J V oice 1999;13(2):303-15. 